\title{
Organization Identifier with Attribute Identifier
}

National Cancer Institute

\section{Source}

National Cancer Institute. Organization Identifier with Attribute Identifier. NCI Thesaurus.

Code C93871.

A unique symbol that establishes identity of the organization. 